conformations. The species barrier might result from the fact that prions that differ at the amino-acid level tend to adopt distinct, non-interacting conformations. But the barrier breaks down if a prion protein is promiscuous and can adopt both conformations.

Raluca Gagescu

(2) References and links original Research paPer Chien, P. \& Weissman, J. S. Conformational diversity in a yeast prion dictates its seeding specificity. Nature 410, 223-227 (2001) FURTHER READING Aguzzi, A. et al. Prions: health scare and biological challenge. Nature Rev. Mol. Cell Biol. 2, 118-126 (2001) S Serio, T. R. \& Lindquist, S. L. [PSI+]: an epigenetic modulator of translation termination efficiency. Annu. Rev. Cell Biol. 15, 661-703 (1999)

ELS LINK Prions

AUTHOR LAB PAGE Weissman laboratory

could be easily fitted into lower-resolution electron microscope data to produce a model for the straight flagella formed by R-type protofilaments alone (as in the figure).

To understand how the R-type protofilament converts into the $\mathrm{L}$ type, the Japanese group used computer modelling to stretch their structure. Three flagellin subunits were taken, arranged as in a protofilament. The end subunits were kept rigid while being pulled apart in $0.1-\AA$ steps, and the central subunit was allowed to relax to its lowest energy state. At first there were no major changes in structure, but then, over a $0.2-\AA$ stretch, the central flagellin underwent a subtle conformational change. A $\beta$-hairpin shifted to allow the $0.8-\AA$ expansion required to lengthen the protofilament.

The bacterial flagellum has been one of the most intensely studied structures in biology, and what has piqued the curiosity of biochemists, biophysicists and engineers alike is its changes between forward swimming and reverse tumbling. At long last we are beginning to see exactly how subthe this switch in gear really is.

Christopher Surridge Senior Editor, Nature

(2) References and links ORIGINAL RESEARCH PAPER Samatey, F. A. et al. Structure of the bacterial flagellar protofilament and implications for a switch for supercoiling Nature 410, 331-337 (2001)

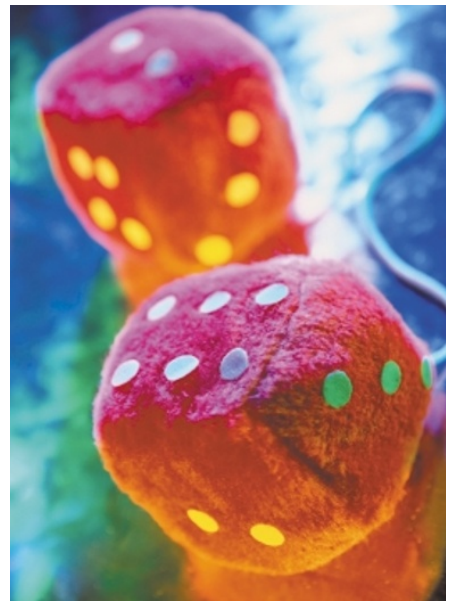

CELL DIVISION

\section{Counting on EGFR}

During development, it is just as crucial to have the right number of cells as it is to specify their fates correctly. Extracellular cues have long been thought to regulate cell number, but what are these cues? A paper in Cell now reveals that, in the Drosophila melanogaster eye, activation of the epidermal growth factor receptor (EGFR) triggers division and that this same signal regulates cell survival.

Baker and $\mathrm{Yu}$ focused on a group of cells that form late in the eye disc. By forming mutant clones within a wild-type tissue, they found that spitz, the ligand for EGFR, is important for division. Looking at different markers for cells that have entered the cell cycle, they then showed that the cells were arrested in G2.

This led them to wonder whether EGFR is required for G2/M progression. As suspected, EGFR mutant clones did not enter mitosis. Using EGFR overexpression, they then confirmed that autonomous EGFR is sufficient to trigger division.

Where does the trigger for EGFR activation come from? The authors showed that neighbouring cells provide this signal — a mechanism that might ensure coordination between the number of cell types in the tissue.

Once the cells are formed, the authors showed, EGFR mediates their survival - a role previously suspected and which is distinct from its function in G2/M progression. So it seems that EGFR has a dual function ensuring that enough cells form and that, once formed, they do not die.

$$
\text { Alison Schuldt }
$$

(2) References and links ORIGINAL RESEARCH PAPER Baker, N. E. \& Yu, S.-Y. The EGF receptor defines domains of cell cycle progression and survival to regulate cell number in the developing Drosophila eye. Cell 104, 699-708 (2001)
H I G H L I G H T S

\section{IN BRIEF}

\section{MOLECULAR MOTORS}

Cargo of kinesin identified as JIP scaffolding proteins and associated signaling molecules.

Verhey, K. J. et al. J. Cell Biol. 152, 959-970 (2001)

We understand in some detail how kinesin moves, but we know little about its cargo. Verhey et al. show that kinesin binds to three scaffolding proteins in the JNK signalling pathway, JIP-1, JIP-2 and JIP-3. Several signalling molecules are present in the complex, indicating that kinesin might transport pre-assembled signalling complexes. Could signalling molecules, in turn, regulate kinesin?

\section{TECHNIQUE}

Delivery of proteins into living cells by reversible membrane permeabilization with streptolysin-O. Walev, I. et al. Proc. Natt Acad. Sci. USA 98, 3185-3190 (2001)

It is not easy to introduce reagents into cells without permanently damaging them. Permeabilization with streptolysin-O - a standard cell-biology technique - might be a solution to this problem. Walev and colleagues report that it is possible to introduce whole proteins into cells permeabilized with low concentrations of streptolysin-O. The cells reseal and remain viable for days in culture, allowing the effects of the introduced proteins (or reagents) to be studied in living cells.

\section{MEMBRANE FUSION}

\section{SNARE complex oligomerization by synaphin/} complexin is essential for synaptic vesicle exocytosis.

Tokumaru, H, et al. Cell 104, 421-432 (2001)

Membrane fusion involves the assembly of SNARE transcomplexes to which VAMPs and syntaxins each contribute one $\alpha$ helix, and SNAP-25 contributes two. Tokumaru et al. propose that synaphin facilitates the assembly of SNARE trans-complexes and the oligomerization of several SNARE complexes into a 'rosette'. One SNAP-25 molecule might take part in two neighbouring SNARE complexes, contributing one helix to each. In this way, several SNARE complexes could be serially connected, and this might increase the speed of fusion at nerve termini.

\section{TRANSLOCATION}

The three modules of ADP/ATP carrier cooperate in receptor recruitment and translocation into mitochondria.

Wiedemann, N. et al. EMBO J. 20, 951-960 (2001)

The translocation of ADP/ATP carriers of the inner mitochondrial membrane is puzzling as, instead of one aminoterminal pre-sequence, they contain targeting information in all three of their modules. Which signal is, then, the most important? Here, the authors report that signals in all three modules cooperate at each stage of the translocation process, including binding to the receptor Tom70, translocation (through loop formation), and dimerization in the inner membrane. 